\title{
Forced exercise attenuates neuropathic pain in chronic constriction injury of male rat: an investigation of oxidative stress and inflammation
}

This article was published in the following Dove Press journal:

Journal of Pain Research

23 June 2017

Number of times this article has been viewed

\author{
Hossein Ali Safakhah ${ }^{1,2}$ \\ Nasroallah Moradi Kor ${ }^{2,3}$ \\ Atiyeh Bazargani ${ }^{3}$ \\ Ahmad Reza Bandegi ${ }^{4}$ \\ Hamid Gholami Pourbadie ${ }^{5}$ \\ Baharak Khoshkholgh-Sima ${ }^{5}$ \\ Ali Ghanbari²
}

'Department of Physiology, Faculty of Medicine, Semnan University of Medical Sciences, Semnan, Iran; ${ }^{2}$ Research Center of Physiology, Faculty of Medicine, Semnan University of Medical Sciences, Semnan, Iran; ${ }^{3}$ Student Research Committee, Faculty of Medicine, Semnan University of Medical Sciences, Semnan, Iran; ${ }^{4}$ Department of Biochemistry, Faculty of Medicine, Semnan University of Medical Sciences, Semnan, Iran; ${ }^{5}$ Department of Physiology and Pharmacology, Pasteur Institute of Iran, Tehran, Iran

Correspondence: Ali Ghanbari Research Center of Physiology, Faculty of Medicine, Semnan University of Medical Sciences, PO Box: 35198-9995I,

Semnan, Iran

Email ghanbari@semums.ac.ir
Background and objective: Initial peripheral/central nerve injuries, such as chronic constriction injury (CCI)/spinal cord injury, are often compounded by secondary mechanisms, including inflammation and oxidative stress, which may lead to chronic neuropathic pain characterized by hyperalgesia or allodynia. On the other hand, exercise as a behavioral and non-pharmacological treatment has been shown to alleviate chronic neuropathic pain. Therefore, this study was conducted to examine whether or not exercise reduces neuropathic pain through modifying oxidative stress and inflammation in chronic constriction injury of the sciatic nerve.

Materials and methods: Wistar male rats weighing $200 \pm 20 \mathrm{~g}$ were randomly divided into five groups (normal, sham, CCI, pre-CCI exercise, and post-CCI exercise group). Sciatic nerve of anesthetized rats was loosely ligated to induce CCI, and they were then housed in separate cages. The rats ran on treadmill at a moderate speed for 3 weeks. Mechanical allodynia and thermal hyperalgesia were determined using von Frey filament and plantar test, respectively. Tumor necrosis factor-alpha (TNF- $\alpha$ ) assayed in the cerebrospinal fluid, malondialdehyde, and total antioxidant capacity were measured in the serum using Western blot test, thiobarbituric acid, and ferric reducing ability of plasma (FRAP), respectively.

Results: The mechanical allodynia $(P=0.024)$ and thermal hyperalgesia $(P=0.002)$ in the CCI group were higher than those in the sham group. Exercise after CCI reduced $(P=0.004)$ mechanical allodynia and thermal hyperalgesia $(P=0.025)$ compared with the CCI group. Moreover, the level of FRAP in the CCI group was $(P=0.001)$ lower than that in the sham group, and post-CCI exercise reversed FRAP amount toward the control level $(P=0.019)$. The amount of malondialdehyde did not differ between groups. Level of TNF- $\alpha$ increased in the CCI group $(P=0.0002)$ compared with sham group and post-CCI exercise could reverse it toward the level of control $(P=0.005)$.

Conclusion: Post CCI-exercise but not pre CCI-exercise reduces CCI-induced neuropathic pain. One of the possible involved mechanisms is increasing the total antioxidant capacity and reducing the amount of TNF- $\alpha$.

Keywords: CCI, TNF- $\alpha$, treadmill exercise, neuropathic pain, oxidative stress

\section{Introduction}

Neuropathic pain is an excruciating form of chronic pain that is produced by initial injuries to peripheral or central nervous systems with a complex pathophysiology. ${ }^{1,2}$ In normal situations, the pain begins when a severely painful or damaging stimulus activates primary pain sensory neurons with high activity threshold. However, the response threshold of nociceptors decreases following the neural injury, and neuropathic pain appears often with allodynia (painful response to non-painful stimuli) and hyperalgesia (increased severity of response to painful stimuli). ${ }^{3}$ 
Neuropathic pain is divided into peripheral and central type, based on the order of injured neurons, the former is related to the injury of the first order of sensory neurons, and the latter is related to the second or third order neurons depending on whether the spinal cord or the brain is injured. ${ }^{4}$ Different animal models have been developed to simulate and thus study the peripheral neuropathy. Most of these models are based on procedures performed on the sciatic nerve. In this study, the chronic constriction injury (CCI) of the sciatic nerve, common method for causing peripheral neuropathy, ${ }^{5}$ was used to simulate the neuropathic pain.

Numerous conditions, including autoimmune diseases (e.g., multiple sclerosis), metabolic disorders (such as diabetes), vascular diseases (e.g., stroke), trauma, and cancer cause neuropathic pain in humans. ${ }^{6}$ The most prevalent neuropathies in humans are diabetic polyneuropathy and neuropathies caused by chronic lumbar pains. ${ }^{7}$ Following peripheral nerve injuries, various mechanisms, including increased expression of voltage-dependent sodium channels in the injured primary sensory neurons and adjacent normal neurons, ${ }^{8,9}$ induced peripheral and central hyper-sensitivity, ${ }^{10,11}$ structural changes, such as neuronal sprouting, ${ }^{8}$ and oxidative stress ${ }^{12}$ lead to neuropathic pain. Oxidative stress plays a critical role in pathogenesis of different conditions, including neurological disorders, spinal cord injury and neuropathic pain. ${ }^{13,14}$ A close relationship between oxidative stress and inflammation has been reported so that oxidative stress following inflammation can induce more inflammation through different pathways and vice versa. ${ }^{15,16}$ Oxidative stress such as protein oxidation and lipids peroxidation causes neuronal damage. ${ }^{17}$ In addition, it has been reported that oxidative stress in diabetes promotes neuronal degeneration through increase of proinflammatory cytokines such as tumor necrosis factor-alpha (TNF- $\alpha$ ) and interleukin (IL)- $6 .{ }^{18}$ On the other hand, TNF- $\alpha$ in turn leads to neuropathic pain by increasing excitability of sensory neurons in pain pathways. ${ }^{19}$ It was suggested that TNF- $\alpha$ increases $\alpha$-amino-3-hydroxy5-methyl-4-isoxazolepropionic acid receptor conductance on pain pathways and therefore decreases threshold activation of nociceptors, which leads to neuronal hyperexcitability and pathologic pain. ${ }^{20}$ Due to the multiplicity of mechanisms involved in neuropathic pain, pharmacological treatments (nonsteroidal anti-inflammatory drugs, opioids, antidepressants, and anticonvulsants) that have been introduced so far could not treat or prevent the spread of pain and caused many side effects as well. ${ }^{21}$ In this respect, using a method with no side effects or with minimum side effects is of great interest.
Exercise, especially regular exercise, as a behavioral and non-pharmacological method has positive effects on health. ${ }^{22,23}$ It has been reported that exercising has positive effects on brain injuries caused by strokes in animal models. ${ }^{24}$ Treadmill exercise, as a non-invasive treatment, has many potential effects on the consequences of spinal cord injury such as pain. The exact mechanism of hypoalgesia after exercise is not known, but it is believed that the activity of endogenous opioid system and release of central and peripheral beta-endorphins ${ }^{25,26}$ play an important role in this phenomenon. Furthermore, other reports reveal that exercise decreases neuropathic pain through reducing the inflammatory factors. ${ }^{27}$ Lopez-Alvarez et al reported that reduced brain-derived neurotrophic factor (BDNF) and nerve-growth factor (NGF) levels in the spinal sensory neurons following exercise, alleviates neuropathic pain in sciatic injured rats. ${ }^{28} \mathrm{~A}$ recent study showed that increased pain and reduced dorsal horn GABAergic tone in sciatic nerve injured mice were significantly inhibited through treadmill exercise. ${ }^{29}$ Numerous studies show that exercise changes the antioxidant capacity and can act as an antioxidant. ${ }^{30,31}$ Regular exercise induces the endogenous antioxidant system, which may protect the body from consequences of injuries caused by the oxidative stress. ${ }^{32}$ Exercise may have a beneficial effect in diabetes, as the most frequent cause of peripheral neuropathy, through reducing blood glucose, intermediate and end products of advanced glycosylation. ${ }^{33}$ Activation of glial cells and glycogen synthase kinase 3 (GSK-3) leads to proinflammatory cytokine release such as TNF- $\alpha$ and IL-1B. On the other hand proinflammatory cytokines are involved in peripheral nerve damage, sensory neuron hyperexcitability and neuropathic pain induction. ${ }^{34}$ Bayod et al reported that exercise reduces GSK-3 activity of hippocampal neurons in rodents. ${ }^{35}$ It has been also reported that exercise through increasing release of systemic norepinephrine inactivates GSK-3B,${ }^{36}$ which in turn reduces microglial cell proliferation and its proinflammatory cytokine release. ${ }^{37}$ Furthermore, it has been reported that regular routine exercise can prevent or at least decreases inflammation. ${ }^{38}$

Regardless of the precise mechanism of exercise in the course of diseases, it is obvious that exercise positively affects different diseases showing its value as a complementary treatment. The review of previous studies showed that the studies performed on effects of exercise in neuropathic conditions focused mostly on the effects of exercise after neuropathy, and there is no report about its effects before neuropathy. Therefore, this study was performed to examine the effects of exercise on pain before neuropathy. 


\section{Materials and methods Animals}

This study was performed on Wistar adult male rats weighing $200 \pm 20 \mathrm{~g}$, with free access to food and water and housed at 12:12 light-dark cycles at fixed temperature of $22^{\circ} \mathrm{C} \pm 2^{\circ} \mathrm{C}$. The study was approved by the Ethics Committee of the Faculty of Medicine, Semnan University of Medical Sciences, under permit number 93/584235. All experimental procedures were performed according to National Institutes of Health guidelines for caring and working with laboratory animals.

\section{Experimental design}

The study design is shown in Table 1.

\section{Procedures of the surgery and induction of neuropathy}

The rats were first anesthetized using intraperitoneal injection of ketamine $(80 \mathrm{mg} / \mathrm{kg})$ and xylazine $(10 \mathrm{mg} / \mathrm{kg})$. Upon being sure of their deep anesthesia, the rats' left thigh was shaved, and a 2-cm incision was made on their thigh using a surgical blade. Once the muscle was cut to expose the sciatic nerve, the connective tissue was cleared off using two small glass bars, and four loose ligations were made on the nerve at one millimeter intervals using catgut chromic sutures 4.0 around the common sciatic nerve. Then, the muscle and the skin were stitched separately using silk sutures $4.0 .{ }^{5}$ All the rats were housed in separate cages for $24 \mathrm{~h}$ after the surgery to recover and begin eating and drinking.

\section{Preparation of the cerebrospinal fluid}

The cerebrospinal fluid was drawn from cisterna magna using a method introduced by Liu and Duff. ${ }^{39}$ To do so, the rats were anesthetized using ketamine $(80 \mathrm{mg} / \mathrm{kg})$ and xylazine $(10 \mathrm{mg} / \mathrm{kg})$. Their necks were shaved, and their heads were fixed in a stereotaxic apparatus at $45^{\circ}$. Once the site was disinfected with betadine, a longitudinal incision was made on the midline from the end of the occipital bone toward the neck, and the surrounding muscles were cleared off. A thin capillary tube with an external diameter of $0.5 \mathrm{~mm}$ was gently inserted in the cisterna magna in order that the transparent cerebrospinal fluid enters the tube. The sample was collected in a microtube and kept at $-80^{\circ} \mathrm{C}$ until TNF- $\alpha$ assay.

\section{Preparation of serum sample}

Once the cerebrospinal fluid was prepared, a blood sample was drawn from the rats' heart while they were still anesthetized. The serum was then removed from the blood using a centrifuge with 2000 RPM for 10 min and kept at $-80^{\circ} \mathrm{C}$ until malondialdehyde (MDA) and ferric reducing ability of plasma (FRAP) assays.

\section{The study groups and exercising protocol}

This study was performed on 45 rats that were randomly divided into five groups (normal, sham, CCI, pre-CCI exercise, and post-CCI exercise group). The rats excluded from the study were as follows: 2 rats during the training stage (familiarity with the treadmill) due to their inability to run on the treadmill, 1 rat due to its resistance to anesthesia, and another rat that was paralyzed after the surgery. Each group consisted of 6-9 rats.

The forced exercise was implemented on a rat treadmill at a moderate speed for 21 days. The rats ran on the treadmill 5 days a week, $30 \mathrm{~min}$ per day, at the speed of $16 \mathrm{~m} / \mathrm{min}$ close to $70 \% \mathrm{VO}_{2} \max$ for 3 weeks. Prior to the main exercising program, the rats ran on the treadmill for 5 days, 10 min per day, at the speed of $10 \mathrm{~m} / \mathrm{min}$ in order to adapt to the conditions of exercise, and rats that were unable to perform the training task were excluded from the study. ${ }^{26}$

\section{The study groups}

Group 1 (normal): Behavioral and biochemical experiments were performed in this group without any intervention.

Group 2 (sham): Once the skin and muscles on the sciatic nerve were cut in this group, the incision site was stitched without manipulating the nerve. Behavioral tests were

Table I Experimental design and groups

\begin{tabular}{|c|c|c|c|c|c|}
\hline Groups & $\begin{array}{l}3 \text { weeks exercise, } \\
\text { days }-21 \text { to } 0\end{array}$ & $\begin{array}{l}\text { Surgery day, } \\
\text { day I }\end{array}$ & $\begin{array}{l}\text { Recovery } \\
\text { time, days 2-4 }\end{array}$ & $\begin{array}{l}3 \text { weeks exercise, } \\
\text { days } 5-25\end{array}$ & Tests, day 26 \\
\hline Intact & - & - & - & - & $\sqrt{ }$ \\
\hline Sham & - & $\sqrt{ }$ & - & - & $\sqrt{ }$ \\
\hline $\mathrm{CCl}$ & - & $\sqrt{ }$ & - & - & $\sqrt{ }$ \\
\hline Pre-CCI Exercise & $\sqrt{ }$ & $\sqrt{ }$ & - & - & $\sqrt{ }$ \\
\hline Post-CCI Exercise & - & $\sqrt{ }$ & $\sqrt{ }$ & $\sqrt{ }$ & $\sqrt{ }$ \\
\hline
\end{tabular}

Abbreviation: $\mathrm{CCl}$ : chronic constriction injury. 
performed in this group 3 weeks after rehabilitation (recovery from surgery and walking without lagging) (3 days). ${ }^{40}$

Group 3 (CCI): Once the skin and muscles on the sciatic nerve were cut in this group, 4 loose stitches were made on the nerve. No exercising intervention was applied in this group and behavioral and biochemical tests were performed 3 weeks after rehabilitation (3 days).

Group 4 (pre-CCI exercise): This group underwent the same conditions in Group 3, except that the rats exercised 3 weeks just before the induction of neuropathy.

Group 5 (post-CCI exercise): The neuropathy was induced in this group as in Group 3. After 3 days of rehabilitation, the exercising program was performed for 3 weeks, and the day after, behavioral and biochemical tests were performed.

\section{Methods for assessment of behavioral responses to the neuropathic pain}

The following tests were used to examine the rats' behavior and find the effects of exercise on their neuropathic pain.

\section{Mechanical allodynia}

The rats were placed on a wired mesh inside a Plexiglas compartment $(20 \times 20 \times 30 \mathrm{~cm})$, and different Von Frey filaments were used to measure the mechanical allodynia after 10 min when the rats adapted to the new environment. These filaments are made in a way that the pressure exerted on the surface does not change with increases in the pressure applied by the experimenter due to their flexibility. Each filament exerts a certain amount of pressure (in grams) on the surface in proportion to its diameter. The filaments used in this study exert 2-60 g of pressure on the surface (Stoelting Company, Wood Dale, IL, USA). The pressure exerted by the filaments ranged $2,4,6,8,10,15,26,60 \mathrm{~g}$, starting from the lowest pressure and continuing to higher pressures respectively if there was no response. Each filament was pressed to the rat's left plantar 3 times at intervals of $5 \mathrm{~s}$, each time for $1 \mathrm{~s}$, and if the rat responded 2 consecutive times (raised its foot), the pressure was determined as the response threshold, and the test was not continued any longer. If a rat did not respond even to the filament 60 , it was considered as the response threshold. ${ }^{41}$

\section{Thermal hyperalgesia}

In this test, the rats were placed in the Plantar Test device, the infrared radiation source was placed under their injured foot, and the radiation began with regulated intensity after the rats adapted to the new environment. When the device was turned on, the ray radiated to the rats' foot plantar surface and the device recorded the start time. If the rats moved their feet because of burning, the apparatus stopped working, and its timer showed the duration of rats' tolerance in seconds. This test was performed 3 times at intervals of $5 \mathrm{~min}$, and the mean of the 3 times was taken as the response. The duration of test was determined as $60 \mathrm{~s}^{5}$

\section{Biochemical tests}

\section{Measurement of MDA}

The MDA was measured using the rat's serum. The lipid peroxidation was measured through the thiobarbituric acid method. The oxidative stress increases peroxidation of unsaturated fatty acids and thus various aldehydes, including MDA, are produced. Therefore, MDA is considered a marker of oxidative stress and reacts with thiobarbituric acid in acid regions at high temperatures. The maximum absorption was evaluated at 535 nm using a spectrophotometer. ${ }^{42}$

\section{Measurement of total antioxidant capacity (TAC)}

The rat's serum was used to measure the FRAP, which is used to determine the TAC. In this test, the antioxidants are measured by reducing the ferric iron. This method is based on the ability of serums to reduce the ions $\mathrm{Fe}^{3+}$ to $\mathrm{Fe}^{2+}$, and the output is a blue complex with the maximum light absorption at wavelength of $593 \mathrm{~nm} .{ }^{43}$

\section{Measurement of TNF- $\alpha$}

The rat's cerebrospinal fluid was used to measure TNF- $\alpha$. The level of TNF- $\alpha$ in the cerebrospinal fluid was measured using the Western blot test introduced by Boneberg and Hartung. ${ }^{44}$ To ensure the identical protein loading for electrophoresis, the concentration of protein in the cerebrospinal fluid was measured using Bradford protein assay. A certain amount of the sample under electrophoresis was placed on $12 \%$ sodium dodecyl sulfate polyacrylamide gel, and the proteins were then transferred to the polyvinylidene difluoride membrane. To quantify the TNF- $\alpha$ level of cerebrospinal fluid, $4 \mathrm{ng}$ of recombinant TNF- $\alpha$ (eBioscience, Vienna, Austria) was loaded on the gel as a standard control. The membrane was blocked for 90 min using 2\% non-fat powder milk (Amersham, EclAdvance ${ }^{\mathrm{TM}}$, Buckinghamshire, UK) and was then incubated using the TNF- $\alpha$ antibody (1/1000 dilution, Invitrogen. Corporation, Camarillo, CA, USA). The membrane was incubated using horseradish peroxidase (HRP) -conjugated secondary antibodies, and the X-ray film was produced within 1 min using chemiluminescence kit. Quantification of results was performed by densitometry scan of the films. Data analysis was done using Image $\mathrm{J}$ software (National Institute of Health, Bethesda, MD, USA). 


\section{Data analysis}

The data were statistically analyzed using the one-way analysis of variance and Tukey's post-hoc test. All data were stated as mean \pm standard deviation of relevant variables, and $P<0.05$ was considered significant. The Graphpad prism 5.0 statistical software (GraphPad, San Diego, CA, USA) was used to analyze the data.

\section{Results}

\section{$\mathrm{CCl}$ induced mechanical allodynia and thermal hyperalgesia were prevented by moderate post- $\mathrm{CCl}$ exercise}

\section{Mechanical allodynia}

Threshold of response to mechanical stimulus decreased in $\mathrm{CCI}$ group compared to sham group $(P=0.024)$. Exercise on treadmill after induction of CCI increased the threshold of response to the mechanical stimulus in contrast to the CCI group ([P=0.004], F [4 and 30] = 8.2). However, exercise before induction of CCI did not make any significant changes in the threshold against that in the CCI group (Figure 1A). There was no difference between intact and sham groups.

\section{Thermal hyperalgesia}

As in the case of mechanical allodynia, thermal hyperalgesia was examined after CCI induction. The CCI group showed a prominent decrease in the threshold of response to the thermal stimulus compared with sham group $(P=0.002)$. The post$\mathrm{CCI}$ exercise group showed an increase in the withdrawal response to the pain caused by the thermal stimulus on the feet against that in the CCI group ( $[P=0.025], \mathrm{F}$ [4 and 28] $=6.02$ ). However, the group exercising before induction of CCI did not show any significant difference in the level of pain caused by the thermal stimulus compared with the CCI group (Figure 1B).

The increased TNF- $\alpha$ in the cerebrospinal fluid due to $\mathrm{CCI}$ was reversed by post-CCI exercise. The measurement of TNF- $\alpha$ cytokine in the cerebrospinal fluid showed a significant difference between the $\mathrm{CCI}$ group and the sham group; the level of TNF- $\alpha$ in the CCI group was higher than that in the sham group $(P=0.0002)$. Exercise after induction of $\mathrm{CCI}$ ( $P=0.005], \mathrm{F}[4$ and 19$]=12.86)$ decreased the TNF- $\alpha$ level against that in the CCI group (Figure 2). As seen in Figure 2, the level of TNF- $\alpha$ (no difference between intact and sham groups) implied that the surgical incision did not influence the expression of cytokine.
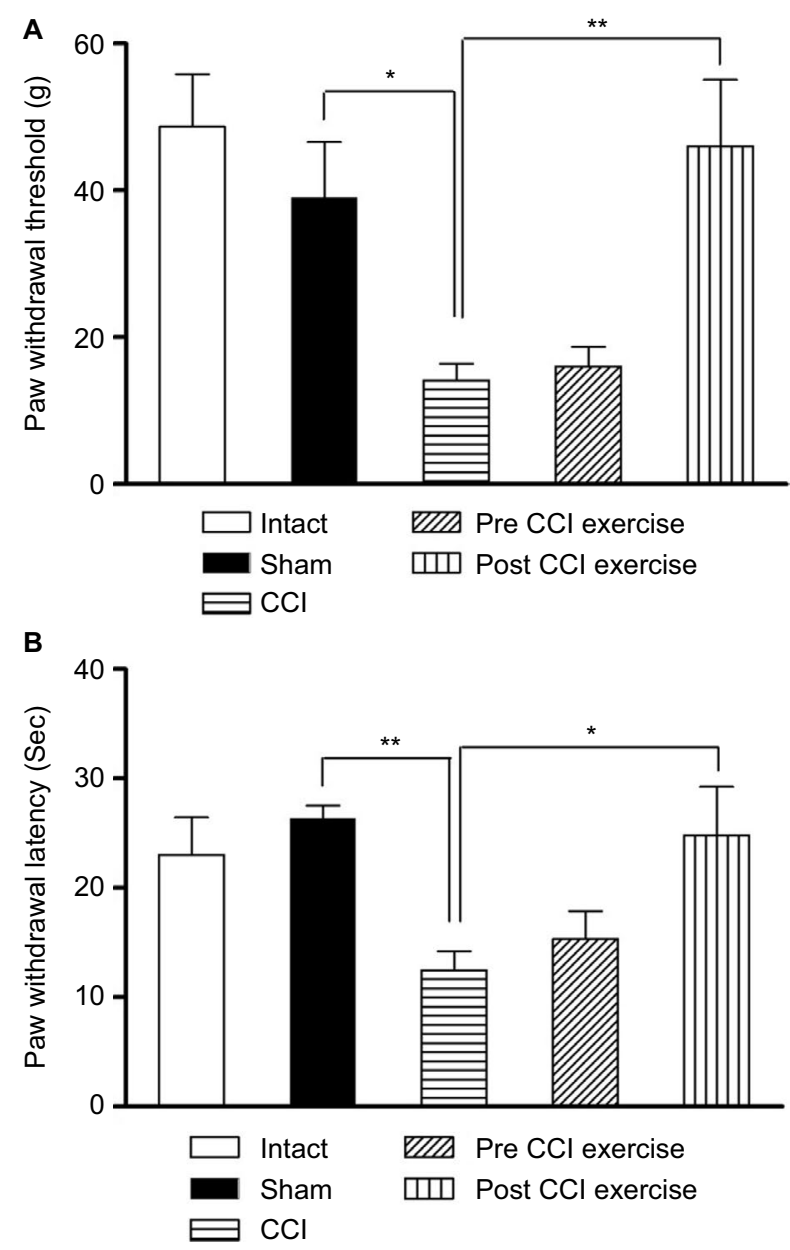

Figure I The effect of exercise on the $\mathrm{CCl}$-induced neuropathic pain. Notes: (A) The threshold of response to the mechanical stimulus in the $\mathrm{CCl}$ group reduced compared with the sham group $(P=0.024)$, and the post- $\mathrm{CCl}$ exercise could reverse the threshold to the control level $(P=0.004)$. (B) The threshold of response to the thermal stimulus in the $\mathrm{CCl}$ group was lower than the sham group $(P=0.002)$. Post $\mathrm{CCl}$ exercise increased the threshold of response $(P=0.025)$ compared with $\mathrm{CCl}$ group. The data are shown as mean \pm SEM. $n=6-8 . * P<0.05, * * P<0.01$.

Abbreviation: $\mathrm{CCl}$, chronic constriction injury.

TAC was decreased by CCI and post-CCI exercise reversed it to the control level. The measurement of serum MDA as an output of the oxidative stress showed no significant difference between post-CCI exercise group and the control group. A similar result was obtained between pre-CCI exercise group and the control group (Figure 3).

The measurement of TAC showed a significant difference between CCI group and the control group; the level of serum antioxidant capacity in the CCI group was lower than the sham group $(P=0.001)$. Exercise after induction of CCI ( $[P=0.019], \mathrm{F}[4$ and 27] $=6.6)$ increased the FRAP level compared with the CCI group, while, exercise before induction of $\mathrm{CCI}$ did not make a significant difference in the level of TAC with respect to the CCI group (Figure 4). 

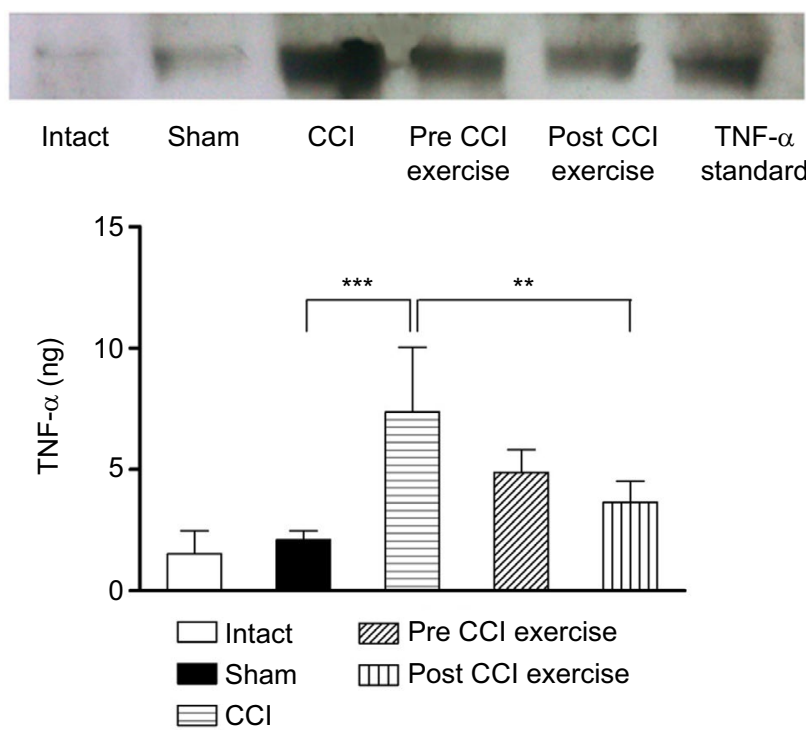

Figure 2 The effect of exercise on the level of TNF- $\alpha$ in the cerebrospinal fluid. Notes: The TNF- $\alpha$ cytokine in the $\mathrm{CCl}$ group was higher than that in the sham group $(P=0.0002)$. The level of this cytokine in the post-CCl exercise group decreased $(P=0.005)$ in contrast to the $\mathrm{CCl}$ group. The data are shown as mean \pm SEM. $\mathrm{n}=4-5$. $* * P<0.01$, $* * * P<0.001$.

Abbreviations: $\mathrm{CCl}$, chronic constriction injury; TNF- $\alpha$, tumor necrosis factoralpha.

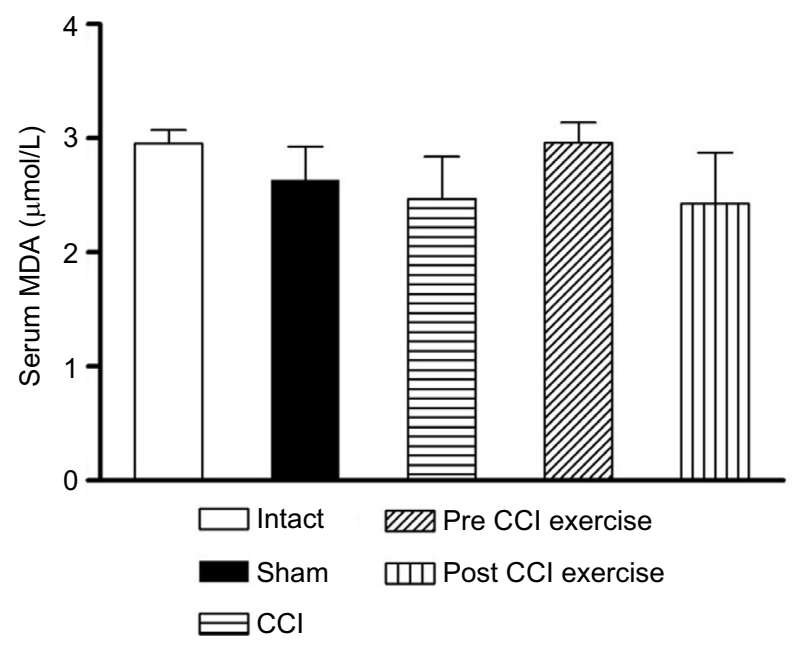

Figure 3 The effect of exercise on the level of serum MDA.

Notes: The level of MDA did not differ significantly among groups. The data are shown as mean \pm SEM. $n=4-9$.

Abbreviations: $\mathrm{CCl}$, chronic constriction injury; MDA, malondialdehyde.

\section{Discussion}

Neuropathic pain appearing after diseases, such as diabetes, cancer, and neural constriction injuries affects patients for a long time forcing them to seek treatment frequently, ${ }^{6,45}$ which imposes high costs on patients and the health system. All the introduced treatments not only impose many side effects on patients but also fail to resolve the pain completely. ${ }^{4,46}$

The results showed that moderate exercise after induction of neuropathy increased the threshold of mechanical pain (decreased mechanical allodynia) and thermal pain.

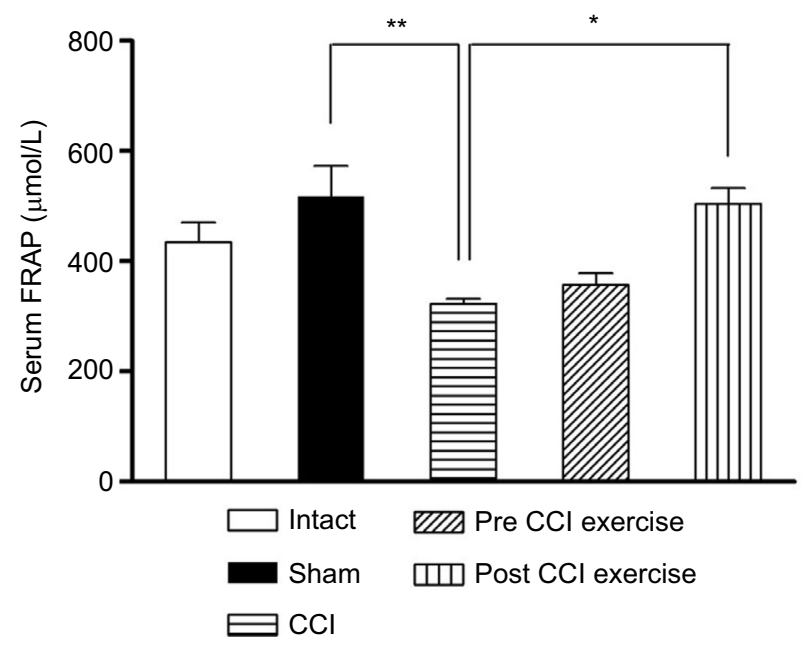

Figure 4 The effect of exercise on the level of serum FRAP.

Notes: The level of serum FRAP in the $\mathrm{CCl}$ group was lower than the sham group $(P=0.00 \mathrm{I})$. The post- $\mathrm{CCl}$ exercise group could increase $(P=0.019)$ the level of serum FRAP compared with the $\mathrm{CCl}$ group. The data are shown as mean \pm SEM. $n=4-9$. $* P<0.05, * * P<0.01$.

Abbreviations: $\mathrm{CCl}$, chronic constriction injury; FRAP, ferric reducing ability of plasma.

The hypoalgesic effect of the physical activity appeared after 3 weeks of running at a moderate speed. This result conformed to results showing that aerobic exercises decrease the mechanical allodynia and thermal hyperalgesia arising from peripheral nerve injuries. ${ }^{47,48}$ Chen et al ${ }^{49}$ also reported that exercise on treadmill decreases mechanical allodynia and the pain caused by the thermal stimulus of CCI in rats. The measurement of TNF- $\alpha$ cytokine in the cerebrospinal fluid showed that the level of TNF- $\alpha$ in the CCI group significantly increased compared with the sham group. In this regard, Okamoto et al indicated that the amount of cytokines increases at the site of injury following the neuropathy caused by the CCI. ${ }^{27}$ Pathologic conditions in the nervous system, such as neuronal injuries and strokes following ischemia, stimulate the microglia and production and release of inflammatory molecules, including cytokines. ${ }^{50}$ The results of our study revealed that exercise on treadmill after induction of neuropathy significantly reduced the TNF- $\alpha$ proinflammatory cytokines compared with the non-exercise neuropathic group (CCI group), and as mentioned before, the neuropathic pain in this group (the post-CCI exercise group) significantly decreased compared with the non-exercise neuropathic group. This result agreed with numerous reports showing that exercise after sciatic injuries reduces the proinflammatory cytokines. ${ }^{49,51}$ Moreover, it has been shown that proinflammatory cytokines, such as TNF- $\alpha$, induce neuropathic pain. ${ }^{52}$ In conformity to this result, Chen et al showed that exercise on treadmill reduced allodynia and hyperalgesia on the 21 st day 
after CCI.$^{49}$ Therefore, it seems that inflammatory cytokines play a remarkable role in the incidence of neuropathic pain, as various studies have shown the correlation between neuropathic pain and proinflammatory cytokines. ${ }^{53-56}$ Since TNF- $\alpha$ can stimulate production of other inflammatory markers such as IL-6, prostanoides, and C-reactive protein which in turn lead to clinical signs of inflammation (pain and fever) and diseases, reduced TNF- $\alpha$ declines other inflammatory mediators, which in turn leads to suppressed clinical manifestation of inflammation and tissue damage. In this respect, one of the mechanisms of exercise in reduction of neuropathic pain is to reduce proinflammatory cytokines.

The results of this study indicated that FRAP, which is an oxidative stress marker, ${ }^{57}$ significantly decreased after CCI. Oxidative stress is a physiological pathway, and its imbalance plays an important role in pathogenesis of neural injuries in different neuropathy models. ${ }^{58}$ Similar to this result, various reports have shown that oxidative stress, which is the imbalance between amount of produced oxidants and amount of endogenous antioxidant power, ${ }^{30}$ plays a role in increasing central sensitization, ${ }^{59}$ especially neuropathic pain. ${ }^{14}$ Di Naso et $\mathrm{al}^{60}$ also reported that the increased production of oxidants or decreased endogenous antioxidant activity significantly influences the diabetic neuropathy.

In this study, the post-CCI exercise significantly increased FRAP up to that in the sham group. Furthermore, the level of MDA, which is another oxidative stress marker, did not show any significant differences among sham, CCI, and exercise groups. Consistent with result obtained in FRAP test, numerous studies have shown that exercise changes the antioxidant capacity. ${ }^{61,62}$ It has been reported that some important elements of the endogenous antioxidant defense, such as superoxide dismutase and glutathione peroxidase, are activated during physical activities, which, particularly at moderate intensity, may act as the most efficient antioxidant. ${ }^{30,31}$ However, contradictory reports indicate that exercise causes oxidative stress, as regular physical activities, such as intense and long exercise, have been shown to cause oxidative stress besides their positive effects on health. ${ }^{63}$ Vina et al showed that aerobic exercises increase free radicals in rats and humans. ${ }^{64}$ Various reports show that MDA as an oxidative stress marker increases after exercise. ${ }^{65-67}$ However, other reports imply that regular moderate exercise reduces the level of $\mathrm{MDA}^{68}$ or does not affect the amount of MDA in the liver, ${ }^{69}$ as in this study no significant change was observed in plasma MDA level. In agreement to present result, Niess et al showed that levels of plasma MDA in trained and untrained people in resting position did not differ significantly from those after exercise. ${ }^{70}$ The contradictions about the effects of exercise on oxidative stress may arise from the use of different samples; different type, intensity, and duration of exercise; different age and sex of the experimental animals. For instance, moderate exercise was practiced in this study, but researchers reporting an increase in oxidative stress following exercise have performed high-intensity exercise for a longer time. Given that both increased antioxidants and decreased TAC are considered oxidative stress markers, the significant decrease in FRAP after CCI in this study implied the assumption that the oxidative stress might be at least one of the mechanisms causing neuropathic pain, and one of the hypoalgesic mechanisms of exercise on neuropathic pain might be dealing with the oxidative stress through improving the TAC. Kim et al's report revealed that the level of reactive oxygen species may increase in neuropathic conditions. ${ }^{71}$ Moreover, it has been reported that glutathione, which is an antioxidant and protects cells from free radicals, significantly decreases in neuropathic conditions. ${ }^{72}$ Varija et al also reported that the decrease in glutathione in the spinal cord and nerves shows increased susceptibility to oxidative stress and hyperalgesia and probable use of glutathione against oxidative stress. ${ }^{13}$ The decrease in FRAP following the neural injury in this study might show that FRAP was used to deal with the antioxidants produced in neuropathic conditions and could keep the level of antioxidants, such as MDA at the same level as that in the control group. The assumption is that other oxidative stress markers, such as superoxide dismutase and glutathione peroxidase, increase after exercise and thus were effective in increasing FRAP. According to the results of this study, oxidative stress and increased level of the TNF- $\alpha$ proinflammatory cytokine occur simultaneously with neuropathic pain, and exercise after neuropathy reduces the pain, oxidative stress, and inflammation. However, the present results cannot properly suggest whether oxidative stress changes the release of the cytokine or inflammatory changes cause oxidative stress, and this is one of the limitations of this study and will be discussed in future. Some reports have shown that oxidative stress stimulates the synthesis of proinflammatory cytokines, such as TNF- $\alpha$, and thus results in the progress of maturation of the adaptive immune system through signaling pathways $\mathrm{NF}-\mathrm{Kb}$ and MAPK. ${ }^{73,74}$ In this respect, oxidative stress was likely to appear before the inflammatory process also in this study. The results of the present study revealed that the pre-CCI exercise with the intensity and duration in this study could not reduce the pain after neural injury. Based on the present data, it seems that failure of pre-CCI exer- 
cise to modify TAC and TNF- $\alpha$ level is the most possible reason for ineffectiveness of exercise on neuropathic pain induced by $\mathrm{CCI}$. Michailidis et al reported that following acute exercise, TAC level significantly increases within $2 \mathrm{~h}$ and returns to pre-exercise level $6 \mathrm{~h}$ later. ${ }^{75}$ Since in our study pain examination was performed 3 weeks post-CCI, it is largely acceptable that effect of pre-CCI exercise on the level of TAC has subsided during this long period of time. In this case, CCI-induced reactive oxygen species production could not be reversed by reduced level of TAC. Eventually, with regard to vicious cycle between oxidative stress and inflammation, ${ }^{15,16}$ pre-CCI exercise was largely unable to alleviate induced neuropathic pain. In general, the results of this study show that exercise before induction of neuropathy under conditions followed in this study cannot reduce the pain after neuropathy. However, increases in duration, intensity, and type of exercise before neuropathy may have significant hypoalgesic effects on neuropathic pain. The results imply that exercise under the same conditions can significantly reduce neuropathic pain and parameters relevant to the oxidative stress and proinflammatory cytokines when practiced after induction of neuropathy.

\section{Conclusion}

The results imply that FRAP decreases and proinflammatory cytokine TNF- $\alpha$ increases during neuropathic pain, and post-neuropathy but not pre-neuropathy exercise as a non-pharmacological method reduces the neuropathic pain by modifying oxidative stress and inflammation.

\section{Acknowledgments}

This study was funded by the Deputy of the Research and Technology of Semnan University of Medical Sciences, and the authors hereby appreciate the Deputy.

\section{Disclosure}

The authors report no conflicts of interest in this work.

\section{References}

1. Jain KK. Current challenges and future prospects in management of neuropathic pain. Expert Rev Neurother. 2008;8(11):1743-1756.

2. Almeida C, DeMaman A, Kusuda R, et al. Exercise therapy normalizes BDNF upregulation and glial hyperactivity in a mouse model of neuropathic pain. Pain. 2015;156(3):504-513.

3. Alzate O, Hussain SR, Goettl VM, et al. Proteomic identification of brainstem cytosolic proteins in a neuropathic pain model. Brain Res Mol Brain Res. 2004;128(2):193-200.

4. Hulsebosch CE. Mechanisms and treatment strategies for chronic central neuropathic pain after spinal cord injury. Top Spinal Cord Inj Rehabil. 2003;8(4):76-91.
5. Bennett GJ, Xie YK. A peripheral mononeuropathy in rat that produces disorders of pain sensation like those seen in man. Pain. 1988;33(1): 87-107.

6. Campbell JN, Meyer RA. Mechanisms of neuropathic pain. Neuron. 2006;52(1):77-92.

7. Nickel FT, Seifert F, Lanz S, Maihofner C. Mechanisms of neuropathic pain. Eur Neuropsychopharmacol. 2012;22(2):81-91.

8. Woolf CJ, Mannion RJ. Neuropathic pain: aetiology, symptoms, mechanisms, and management. Lancet. 1999;353(9168):1959-1964.

9. Boucher TJ, Okuse K, Bennett DL, Munson JB, Wood JN, McMahon SB. Potent analgesic effects of GDNF in neuropathic pain states. Science. 2000;290(5489):124-127.

10. Ibrahim MM, Deng $\mathrm{H}$, Zvonok A, et al. Activation of $\mathrm{CB} 2$ cannabinoid receptors by AM1241 inhibits experimental neuropathic pain: pain inhibition by receptors not present in the CNS. Proc Natl Acad Sci USA. 2003;100(18):10529-10533.

11. James NC, M RA. Mechanisms of Neuropathic Pain. Neuron. 2006;52:77-92.

12. Negi G, Kumar A, Joshi RP, Sharma SS. Oxidative stress and Nrf2 in the pathophysiology of diabetic neuropathy: old perspective with a new angle. Biochem Biophys Res Commun. 2011;408(1):1-5.

13. Varija D, Kumar KP, Reddy KP, Reddy VK. Prolonged constriction of sciatic nerve affecting oxidative stressors \& antioxidant enzymes in rat. Indian J Med Res. 2009;129(5):587-592.

14. Little JW, Doyle T, Salvemini D. Reactive nitroxidative species and nociceptive processing: determining the roles for nitric oxide, superoxide, and peroxynitrite in pain. Amino Acids. 2012;42(1):75-94.

15. Flohe L, Brigelius-Flohe R, Saliou C, Traber MG, Packer L. Redox regulation of NF-kappa B activation. Free Radic Biol Med. 1997;22(6):1115-1126.

16. Vaziri ND, Rodriguez-Iturbe B. Mechanisms of disease: oxidative stress and inflammation in the pathogenesis of hypertension. Nat Clin Pract Nephrol. 2006;2(10):582-593.

17. Popa-Wagner A, Mitran S, Sivanesan S, Chang E, Buga AM. ROS and brain diseases: the good, the bad, and the ugly. Oxid Med Cell Longev. 2013;2013:963520.

18. Prabhakar O. Cerebroprotective effect of resveratrol through antioxidant and anti-inflammatory effects in diabetic rats. Naunyn Schmiedebergs Arch Pharmacol. 2013;386(8):705-710.

19. Wang XM, Lehky TJ, Brell JM, Dorsey SG. Discovering cytokines as targets for chemotherapy-induced painful peripheral neuropathy. Cytokine. 2012;59(1):3-9.

20. Beattie EC, Stellwagen D, Morishita W, et al. Control of synaptic strength by glial TNFalpha. Science. 2002;295(5563):2282-2285.

21. Kingery WS. A critical review of controlled clinical trials for peripheral neuropathic pain and complex regional pain syndromes. Pain. 1997;73(2):123-139.

22. Pagnussat AS, Michaelsen SM, Achaval M, et al. Effect of skilled and unskilled training on nerve regeneration and functional recovery. Braz J Med Biol Res. 2012;45(8):753-762.

23. Zogaib FG, Monte-Alto-Costa A. Moderate intensity physical training accelerates healing of full-thickness wounds in mice. Braz J Med Biol Res. 2011;44(10):1025-1035.

24. Chen YW, Chen SH, Chou W, Lo YM, Hung CH, Lin MT. Exercise pretraining protects against cerebral ischaemia induced by heat stroke in rats. Br J Sports Med. 2007;41(9):597-602.

25. Bement MK, Sluka KA. Low-intensity exercise reverses chronic muscle pain in the rat in a naloxone-dependent manner. Arch Phys Med Rehabil. 2005;86(9):1736-1740.

26. Stagg NJ, Mata HP, Ibrahim MM, et al. Regular exercise reverses sensory hypersensitivity in a rat neuropathic pain model: role of endogenous opioids. Anesthesiology. 2011;114(4):940-948.

27. Okamoto K, Martin DP, Schmelzer JD, Mitsui Y, Low PA. Pro- and anti-inflammatory cytokine gene expression in rat sciatic nerve chronic constriction injury model of neuropathic pain. Exp Neurol. 2001;169(2):386-391. 
28. Lopez-Alvarez VM, Modol L, Navarro X, Cobianchi S. Early increasingintensity treadmill exercise reduces neuropathic pain by preventing nociceptor collateral sprouting and disruption of chloride cotransporters homeostasis after peripheral nerve injury. Pain. 2015;156(9): 1812-1825.

29. Kami K, Taguchi Ms S, Tajima F, Senba E. Improvements in impaired GABA and GAD65/67 production in the spinal dorsal horn contribute to exercise-induced hypoalgesia in a mouse model of neuropathic pain. Mol Pain. 2016;12.

30. Ji LL. Exercise-induced modulation of antioxidant defense. Ann NY Acad Sci. 2002;959:82-92.

31. Ji LL, Gomez-Cabrera MC, Vina J. Exercise and hormesis: activation of cellular antioxidant signaling pathway. Ann N Y Acad Sci. 2006;1067:425-435.

32. Belviranlı M, Gökbel H. Acute exercise induced oxidative stress and antioxidant changes. ER J Gen Med. 2006;3(3):126-131.

33. Ahn S, Song R. Effects of Tai Chi Exercise on glucose control, neuropathy scores, balance, and quality of life in patients with type 2 diabetes and neuropathy. J Altern Complement Med. 2012;18(12):1172-1178.

34. Chertoff M, Di Paolo N, Schoeneberg A, et al. Neuroprotective and neurodegenerative effects of the chronic expression of tumor necrosis factor alpha in the nigrostriatal dopaminergic circuit of adult mice. Exp Neurol. 2011;227(2):237-251.

35. Bayod S, Del Valle J, Canudas AM, et al. Long-term treadmill exercise induces neuroprotective molecular changes in rat brain. J Appl Physiol (1985). 2011;111(5):1380-1390.

36. Ballou LM, Tian PY, Lin HY, Jiang YP, Lin RZ. Dual regulation of glycogen synthase kinase-3beta by the alpha1A-adrenergic receptor J Biol Chem. 2001;276(44):40910-40916.

37. O’Donnell J, Zeppenfeld D, McConnell E, Pena S, Nedergaard M Norepinephrine: a neuromodulator that boosts the function of multiple cell types to optimize CNS performance. Neurochem Res. 2012;37(11): 2496-2512.

38. Irwin MR, Olmstead R. Mitigating cellular inflammation in older adults a randomized controlled trial of Tai Chi Chih. Am J Geriatr Psychiatry. 2012;20(9):764-772.

39. Liu L, Duff K. A technique for serial collection of cerebrospinal fluid from the cisterna magna in mouse. JVis Exp. 2008;(21).

40. Gaffuri J, Meireles A, Rocha BP, et al. Physical exercise assessment as an analgesia factor in a sciatica experimental model. Revista Brasileira de Medicina do Esporte. 2011;17(2):115-118.

41. Chaplan SR, Bach FW, Pogrel JW, Chung JM, Yaksh TL. Quantitative assessment of tactile allodynia in the rat paw. $J$ Neurosci Methods. 1994;53(1):55-63.

42. Mihara M, Uchiyama M. Determination of malonaldehyde precursor in tissues by thiobarbituric acid test. Anal Biochem. 1978;86(1):271-278.

43. Benzie IF, Strain JJ. The ferric reducing ability of plasma (FRAP) as a measure of "antioxidant power": the FRAP assay. Anal Biochem. 1996;239(1):70-76.

44. Boneberg EM, Hartung T. Granulocyte colony-stimulating factor attenuates LPS-stimulated IL-1beta release via suppressed processing of proIL-1beta, whereas TNF-alpha release is inhibited on the level of proTNF-alpha formation. Eur J Immunol. 2002;32(6):1717-1725.

45. Ueda H. Molecular mechanisms of neuropathic pain-phenotypic switch and initiation mechanisms. Pharmacol Ther. 2006;109(1-2):57-77.

46. Gosselin RD, Suter MR, Ji RR, Decosterd I. Glial cells and chronic pain. Neuroscientist. 2010;16(5):519-531.

47. Cobianchi S, Casals-Diaz L, Jaramillo J, Navarro X. Differential effects of activity dependent treatments on axonal regeneration and neuropathic pain after peripheral nerve injury. Exp Neurol. 2013;240:157-167.

48. Kami K, Taguchi S, Tajima F, Senba E. Histone acetylation in microglia contributes to exercise-induced hypoalgesia in neuropathic pain model mice. J Pain. 2016;17(5):588-599.

49. Chen YW, Li YT, Chen YC, Li ZY, Hung CH. Exercise training attenuates neuropathic pain and cytokine expression after chronic constriction injury of rat sciatic nerve. Anesth Analg. 2012;114(6):1330-1337.
50. Nilupul Perera M, Ma HK, Arakawa S, et al. Inflammation following stroke. J Clin Neurosci. 2006;13(1):1-8.

51. Ramesh G, MacLean AG, Philipp MT. Cytokines and chemokines at the crossroads of neuroinflammation, neurodegeneration, and neuropathic pain. Mediators Inflamm. 2013;2013:480739.

52. Zelenka M, Schafers M, Sommer C. Intraneural injection of interleukinlbeta and tumor necrosis factor-alpha into rat sciatic nerve at physiological doses induces signs of neuropathic pain. Pain. 2005;116(3): 257-263.

53. Clark AK, Old EA, Malcangio M. Neuropathic pain and cytokines: current perspectives. J Pain Res. 2013;6:803-814.

54. Ishikawa T, Miyagi M, Kamoda H, et al. Differences between tumor necrosis factor-alpha receptors types 1 and 2 in the modulation of spinal glial cell activation and mechanical allodynia in a rat sciatic nerve injury model. Spine (Phila Pa 1976). 2013;38(1):11-16.

55. Marchand F, Tsantoulas C, Singh D, et al. Effects of Etanercept and Minocycline in a rat model of spinal cord injury. Eur J Pain. 2009;13(7):673-681.

56. Yoon H, Thakur V, Isham D, Fayad M, Chattopadhyay M. Moderate exercise training attenuates inflammatory mediators in DRG of Type 1 diabetic rats. Exp Neurol. 2015;267:107-114.

57. Urso ML, Clarkson PM. Oxidative stress, exercise, and antioxidant supplementation. Toxicology. 2003;189(1-2):41-54.

58. Negi G, Kumar A, Sharma SS. Melatonin modulates neuroinflammation and oxidative stress in experimental diabetic neuropathy: effects on NF-kappaB and Nrf2 cascades. J Pineal Res. 2011;50(2): $124-131$.

59. Janes K, Neumann WL, Salvemini D. Anti-superoxide and antiperoxynitrite strategies in pain suppression. Biochim Biophys Acta. 2012;1822(5):815-821.

60. Di Naso FC, Simoes Dias A, Porawski M, Marroni NA. Exogenous superoxide dismutase: action on liver oxidative stress in animals with streptozotocin-induced diabetes. Exp Diabetes Res. 2011;2011: 754132 .

61. Shin YA, Lee JH, Song W, Jun TW. Exercise training improves the antioxidant enzyme activity with no changes of telomere length. Mech Ageing Dev. 2008;129(5):254-260.

62. Berzosa C, Cebrian I, Fuentes-Broto L, et al. Acute exercise increases plasma total antioxidant status and antioxidant enzyme activities in untrained men. J Biomed Biotechnol. 2011;2011:540458.

63. Powers SK, Nelson WB, Hudson MB. Exercise-induced oxidative stress in humans: cause and consequences. Free Radic Biol Med. 2011;51(5):942-950.

64. Vina J, Gimeno A, Sastre J, et al. Mechanism of free radical production in exhaustive exercise in humans and rats; role of xanthine oxidase and protection by allopurinol. IUBMB Life. 2000;49(6):539-544.

65. Maughan RJ, Donnelly AE, Gleeson M, Whiting PH, Walker KA, Clough PJ. Delayed-onset muscle damage and lipid peroxidation in man after a downhill run. Muscle Nerve. 1989;12(4):332-336.

66. Noroozi J, Zeynali F, Nematbakhsh M, Pezeshki Z, Talebi A. Nonpreventive role of aerobic exercise against cisplatin-induced nephrotoxicity in female rats. Int J Prev Med. 2015;6:58.

67. Santos-Silva A, Rebelo MI, Castro EM, et al. Leukocyte activation, erythrocyte damage, lipid profile and oxidative stress imposed by high competition physical exercise in adolescents. Clin Chim Acta. 2001;306(1-2):119-126

68. Naziroglu M, Simsek M, Kutlu M. Moderate exercise with a dietary vitamin $\mathrm{C}$ and $\mathrm{E}$ combination protects against streptozotocin-induced oxidative damage to the blood and improves fetal outcomes in pregnant rats. Clin Chem Lab Med. 2004;42(5):511-517.

69. Choi EY, Cho YO. The effects of physical training on antioxidative status under exercise-induced oxidative stress. Nutr Res Pract. 2007;1(1):14-18.

70. Niess AM, Hartmann A, Grunert-Fuchs M, Poch B, Speit G. DNA damage after exhaustive treadmill running in trained and untrained men. Int J Sports Med. 1996;17(6):397-403. 
71. Kim HK, Kim JH, Gao X, et al. Analgesic effect of vitamin E is mediated by reducing central sensitization in neuropathic pain. Pain. 2006;122(1-2):53-62.

72. Struzynska L, Chalimoniuk M, Sulkowski G. The role of astroglia in $\mathrm{Pb}$-exposed adult rat brain with respect to glutamate toxicity. Toxicology. 2005;212(2-3):185-194.

73. Sklavos MM, Tse HM, Piganelli JD. Redox modulation inhibits CD8 T cell effector function. Free Radic Biol Med. 2008;45(10):1477-1486.
74. Hubert MT, Milton MJ, Schreiner S, Profozich JL, Trucco M, Piganelli JD. Disruption of innate-mediated proinflammatory cytokine and reactive oxygen species third signal leads to antigen-specific hyporesponsiveness. J Immunol. 2007;178(2):908-917.

75. Michailidis Y, Jamurtas AZ, Nikolaidis MG, et al. Sampling time is crucial for measurement of aerobic exercise-induced oxidative stress. Med Sci Sports Exerc. 2007;39(7):1107-1113.

\section{Publish your work in this journal}

The Journal of Pain Research is an international, peer reviewed, open access, online journal that welcomes laboratory and clinical findings in the fields of pain research and the prevention and management of pain. Original research, reviews, symposium reports, hypothesis formation and commentaries are all considered for publication.
Dovepress

The manuscript management system is completely online and includes a very quick and fair peer-review system, which is all easy to use. Visit http://www.dovepress.com/testimonials.php to read real quotes from published authors. 